Research Article

\title{
The Electronic Structures and Optical Properties of Electron Tuned Fe-Doped $\mathrm{SnO}_{2}$ Materials
}

\author{
Zhe Wang, Bao-Jun Huang, Chang-Wen Zhang, Xi-Jin Xu, and Pei-Ji Wang \\ School of Physics, University of Jinan, Jinan 250022, China \\ Correspondence should be addressed to Pei-Ji Wang; ss_wangpj@ujn.edu.cn
}

Received 21 July 2014; Accepted 14 August 2014

Academic Editor: Xiang Wu

Copyright (C) 2015 Zhe Wang et al. This is an open access article distributed under the Creative Commons Attribution License, which permits unrestricted use, distribution, and reproduction in any medium, provided the original work is properly cited.

By means of the full-potential linearized augmented plane-wave method (FP-LAPW), the electronic structures and optical properties of $\mathrm{Sn}_{15} \mathrm{FeO}_{32}$ with electron-injection are studied. The results show that Fe-doped $\mathrm{SnO}_{2}$ materials are all direct transition semiconductors. The Fermi level goes into conduction band gradually and the band gap decreases with the increase of electron injection. The peaks of optical properties, such as the imaginary part of dielectric function and absorption spectra, change greatly at low energy. The absorption spectra exhibit blue shift, and the optical absorption edge increases, which are consistent with the change of the band gaps.

\section{Introduction}

The diluted magnetic semiconductors (DMS) have attracted a lot of experimental and theoretical attention [1-4] because their spin and charges can be manipulated, which will hereby induce many interestingly magnetic and magnetooptic characteristics. Though the magnetic and electronic properties in some typical DMS systems, such as $\mathrm{SnO}_{2}, \mathrm{ZnO}$, and $\mathrm{GaN}$, have been investigated extensively, many challenges to realize DMS materials for practical applications [5-7] still exist.

As a wide band-gap semiconductor, doped $\mathrm{SnO}_{2}$ play a promising role in short-wavelength LED, gas sensor, and laser diode due to its large band gap $(3.6 \mathrm{eV})$ and high exciton binding energy $(130 \mathrm{meV})$ at the room temperature [8-10]. The electronic structures, magnetic, and optical properties of transition metal (Co, Cr, Mo, Eu, etc.) doped $\mathrm{SnO}_{2}$ bulk semiconductors materials have been researched in theory and experiment [11-13]. As the important one of the family, the structures and optical properties of Fe-doped $\mathrm{SnO}_{2}$ have also caused much attention [14-16]. Adhikari et al. [17] fabricated Fe-doped $\mathrm{SnO}_{2}$ nanoparticles with a chemical coprecipitation method and studied their structures and magnetism. Kim et al. [18] studied the structure, magnetic and optical properties, and Hall effects of Co- and $\mathrm{Fe}$-doped $\mathrm{SnO}_{2}$. When $\mathrm{Fe}$ doped $\mathrm{SnO}_{2}$, for charge balance, $\mathrm{Fe}$ ions must have the ionic valence of $\mathrm{Fe}^{4+}$ without introducing any defects [19]. But Fe ions do not have 4+ oxidation state; hence, the holes are created. So the electronic injection is necessary to obtain the better performance of $\mathrm{SnO}_{2}$. It is well known that the injection of electrons into semiconductors is indispensable to realize spin-related devices, such as spin transistors [20]. The bleach in the absorption spectra by using size-dependent electron injection from excited CdSe quantum dots into $\mathrm{TiO}_{2}$ nanoparticles is observed. It can be theoretically predicted using the first principles calculation, even if there are many difficulties such as the room temperature and external magnetic field in experiment $[21,22]$. In this work, first-principles spin polarized calculations were used to explore the electron injection into $\mathrm{Fe}$-doped $\mathrm{SnO}_{2}$, and its optical and magnetic properties were studied in order to understand the transition mechanism.

\section{Computational Details}

The first-principle calculations are performed using FPLAPW as implemented in WIEN2k code $[23,24]$. The exchange and correlation effects are treated with the generalized gradient approximations (GGA) $[25,26]$. The parameter of $R_{\mathrm{mt}} K_{\max }$ is chosen to be seven $\left(R_{\mathrm{mt}}\right.$ is the smallest muffintin radius in the unit cell and $K_{\max }$ is the cut-off for the plane 


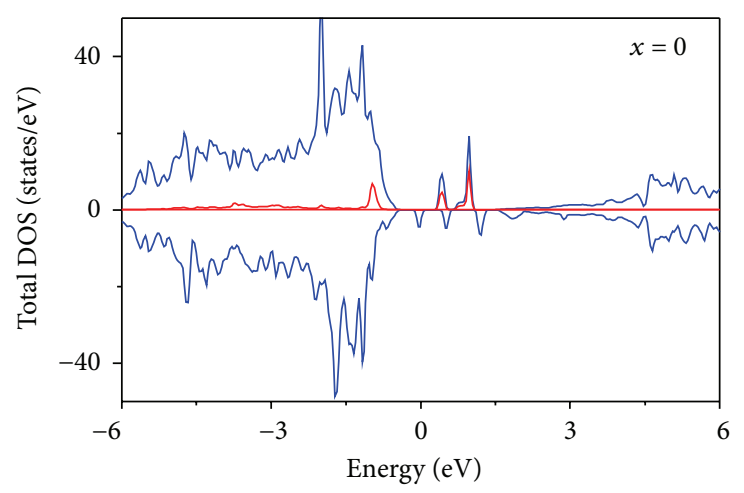

(a)

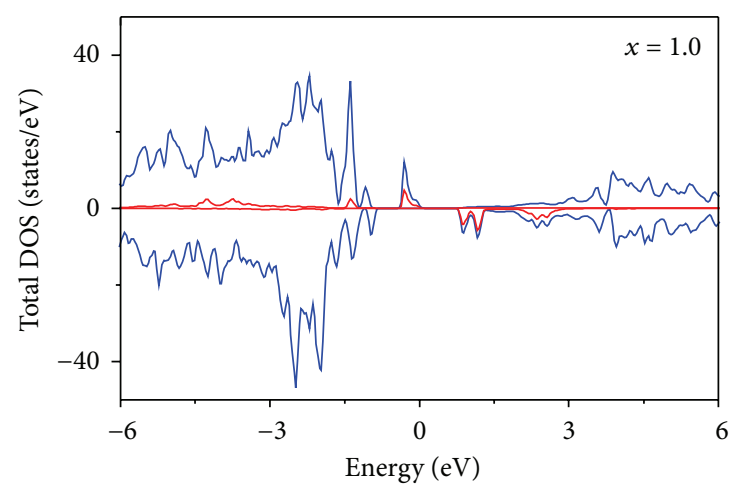

(c)

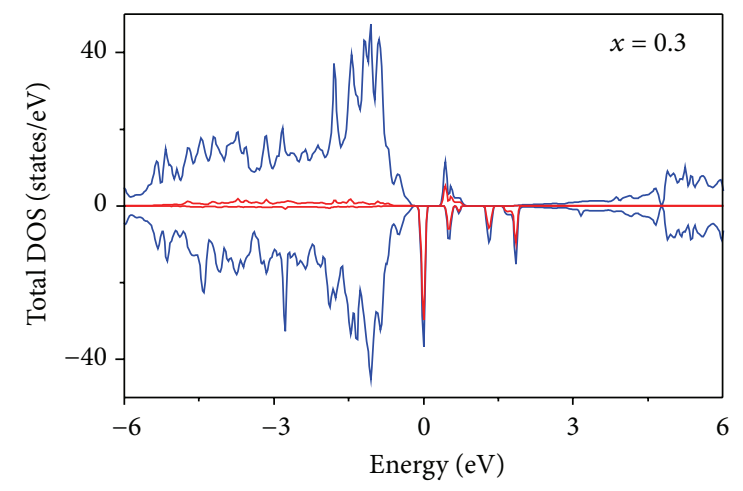

(b)

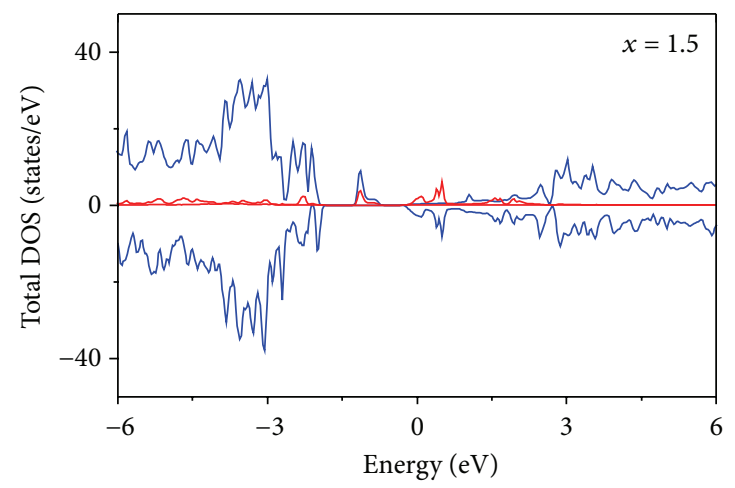

(d)

Figure 1: (Color online) The total DOS of the $\mathrm{Sn}_{15} \mathrm{FeO}_{32}$ supercell. The blue lines represent total DOS and the red lines represent Fe total DOS, respectively. (a) $x=0$, (b) $x=0.3$, (c) $x=1.0$, and (d) $x=1.5$.

wave). The cut-off energy required in the calculations of the solid state is 0.0001 Ry. For $k$-space integration, a grid of 4 $\times 3 \times 3 k$ points in the first Brillouin zone is used. Atomic sphere radii of $\mathrm{Sn}, \mathrm{O}$, and $\mathrm{Fe}$ atoms are set to be 2.0, 1.8, and 2.0 a.u, respectively. The lattice parameters of $\mathrm{SnO}_{2}$ crystals are consistent with the experimental values, which are $a=b$ $=0.4737 \mathrm{~nm}, c=0.3186 \mathrm{~nm}$, and $\alpha=\beta=\gamma=90^{\circ}$ [27].

All calculation models are constructed with $2 \times 2 \times 2$ supercell of $\mathrm{SnO}_{2}$, which contains $16 \mathrm{Sn}$ atoms and $32 \mathrm{O}$ atoms. In current work, only substitutional doping of $\mathrm{Fe}$ with $\mathrm{Sn}$ atoms is considered. Then, electron injection into $\mathrm{Sn}_{15} \mathrm{FeO}_{32}$ is taken with electron concentrations of $x=0,0.3$, $0.5,1.0,1.2$, and 1.5 , respectively, and $x$ is electron injection concentrations. The valence states for $\mathrm{Sn}, \mathrm{O}$, are Fe are $5 \mathrm{~s}^{2} 5 \mathrm{p}^{2}$, $2 s^{2} 2 p^{4}$, and $3 d^{6} 4 s^{2}$, respectively.

\section{Results and Discussion}

\subsection{Electronic Structure}

3.1.1. Density of States (DOS). The calculated total DOS of the $\mathrm{Sn}_{15} \mathrm{FeO}_{32}$ supercell is shown in Figure 1. It can be seen that Fe substitutions into $\mathrm{SnO}_{2}$ DMS induce exchange-split impurity states in the band gap, and the size of impurity states increases with the increase of injected electrons. When the concentration of injected electrons is less than 1.0, the material shows a half-metallic behavior with the majority spin being semiconducting and the minority spin being metallic with sufficient unfilled states above the Fermi level. The $100 \%$ spin polarization carriers suggest that electroninjected Fe-doped $\mathrm{SnO}_{2}$ can be used for spin injection where highly polarized spin current is desired. With the increase of the injected electrons, the conduction band moves to valence band gradually. Besides that, the DOS near the Fermi level reverses when the injected electron is 1.0. When the concentration of injected electrons is large than 1.0, the material shows a metallic behavior with both the majority spin and minority spin across the Fermi level.

The total and partial DOS of $\mathrm{Sn}_{15} \mathrm{FeO}_{32}$ supercell with electron-injection concentration $x=0$ and 1.0 are presented in Figure 2, respectively. The coupling effect of Fe d, O p, and $\mathrm{Sn} \mathrm{p}$ states can be found after Fe doping from Figure 2 . The impurity states in the band gap are mostly composed of Fe $3 \mathrm{~d}$ state hybridized with the O $2 \mathrm{p}$ states. The DOS (Figure 2(b)) indicates that the exchange-split Fe 3d states strongly hybridize with the $\mathrm{O} 2 \mathrm{p}$ states at the top of the valence band, which is partly spin-polarized. When electroninjection concentration is 1.0, the partial DOS of Fe atom is greatly changed, especially in the Fermi level. The lowermost valence bands largely derive from Fe 3d, O 2p, and Sn $5 p$ state when the band ranges from -6.0 to $-3.5 \mathrm{eV}$. The $\mathrm{O} 2 \mathrm{p}$ state and $\mathrm{Fe} 3 \mathrm{~d}$ state are formed when the bands are between -3.5 and $-0.7 \mathrm{eV}$. The states derive from $\mathrm{Fe} 3 \mathrm{~d}$ when the bands are between -0.7 and $0.3 \mathrm{eV}$. However, the states derive from $\mathrm{Fe}$ 


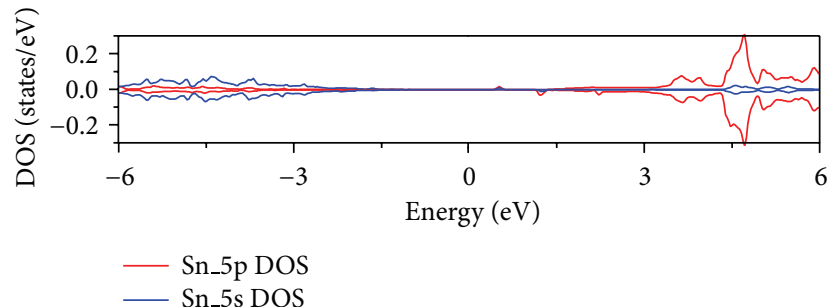

(A)

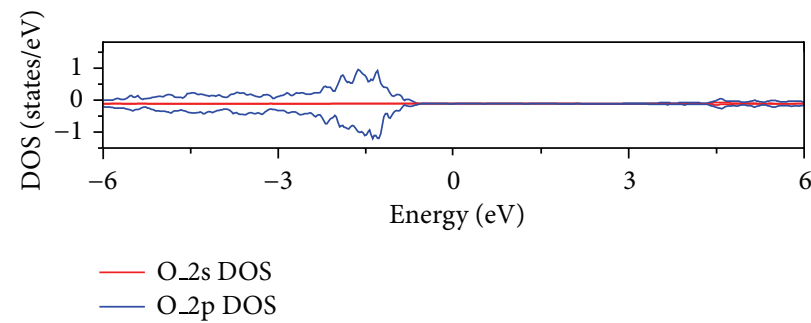

(B)

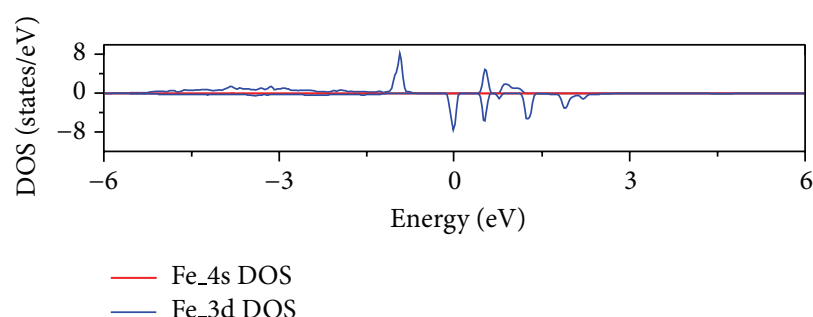

(C)

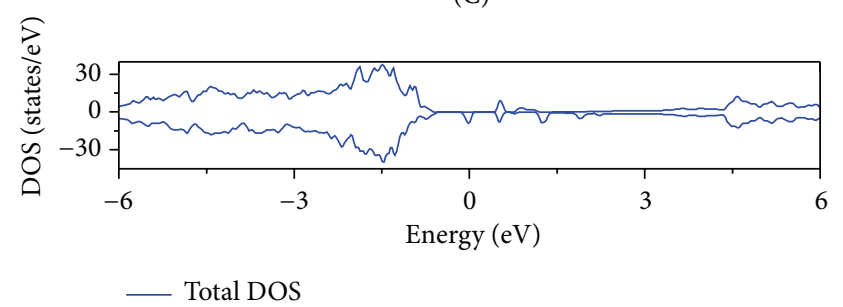

(D)

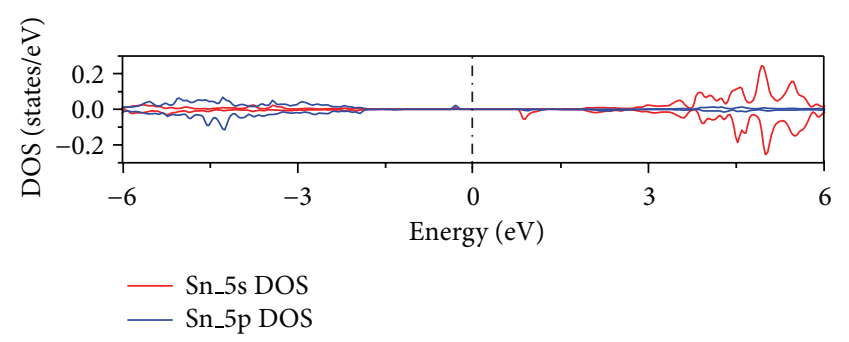

(A)

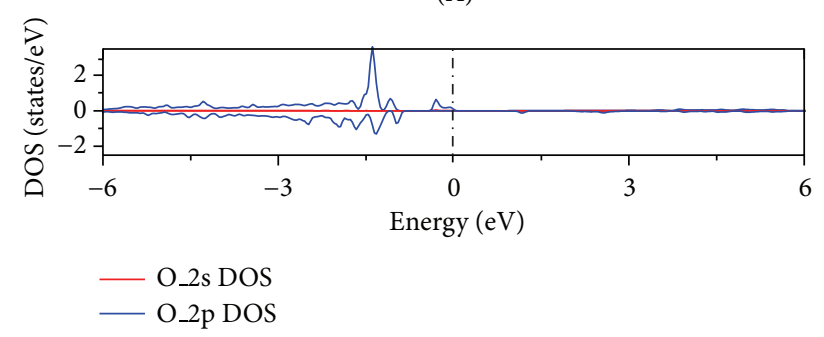

(B)

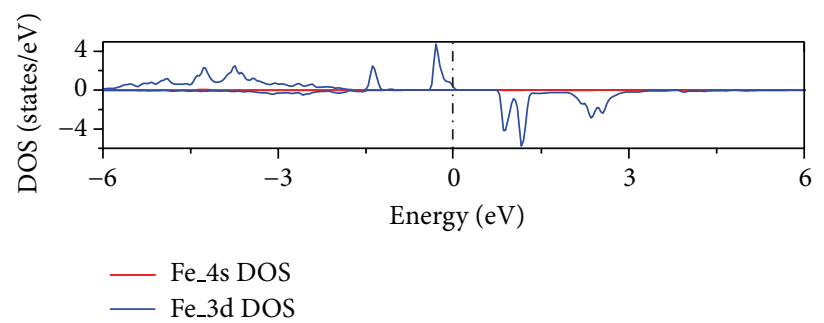

(C)

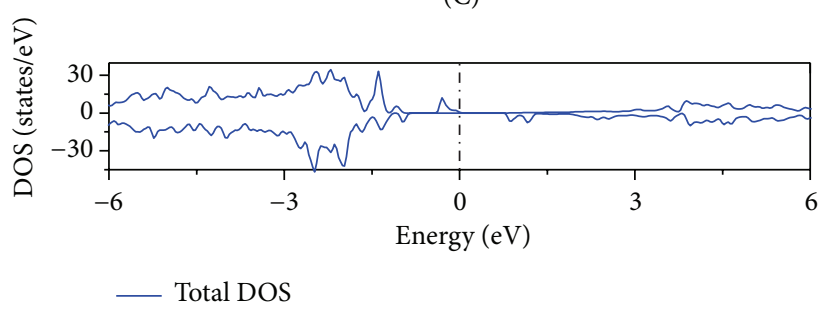

(D)

(a)

(b)

FIGURE 2: (Color online) Total DOS plots and partial DOS plots of the $\mathrm{Sn}_{15} \mathrm{FeO}_{32}$ supercell with electron-injection (1) $x=0$ and (2) $x=1.0$.

$3 \mathrm{~d}$ and $\mathrm{O} 2 \mathrm{p}$ when electron-injection concentration is 1.0 , and Fe $3 d$ turns to down from spin up, indicating that particles reverse has happened. When the bands are in the range from 0.3 to $2.6 \mathrm{eV}$, the states mainly derive from Fe $3 \mathrm{~d}$. When the bands locate in the range from 2.6 to $6.0 \mathrm{eV}$, the states mainly derive from $\mathrm{Sn} 5 \mathrm{p}$.

3.1.2. Band Structure. The band structure of $\mathrm{Sn}_{15} \mathrm{FeO}_{32}$ supercell is shown in Figure 3, when $x=0.5$. At the Fermi level, occupied and not occupied electrons exist when spin down and up, respectively. Figure 4 shows the band structures of spin-up $\mathrm{Sn}_{15} \mathrm{FeO}_{32}$ with the concentration of inject electrons to be 1.0 and 1.5. Furthermore the band gaps become narrower and narrower until zero with the increase of the injected electrons, indicating the excellent conductivity. Each band structure displays a direct band gap at the highly symmetric $\mathrm{G}$ point as well as in pure $\mathrm{SnO}_{2}$, as shown in [28].

3.2. Optical Properties. It is well known that the interaction of a photon with the electrons can be described according to time-dependent perturbations of the ground-state electronic states, and the optical transitions between occupied and unoccupied states are caused by the electric field of the photon. More importantly, solid dielectric function reflects the information between energy band structure and optical spectral lines and can characterize the physical properties of materials. The formula of dielectric function is defined by

$$
\varepsilon(\omega)=\varepsilon_{1}(\omega)+i \varepsilon_{2}(\omega),
$$

where $\varepsilon_{1}(\omega)$ is the real part of the function, while $\varepsilon_{2}(\omega)$ is the imaginary part. 


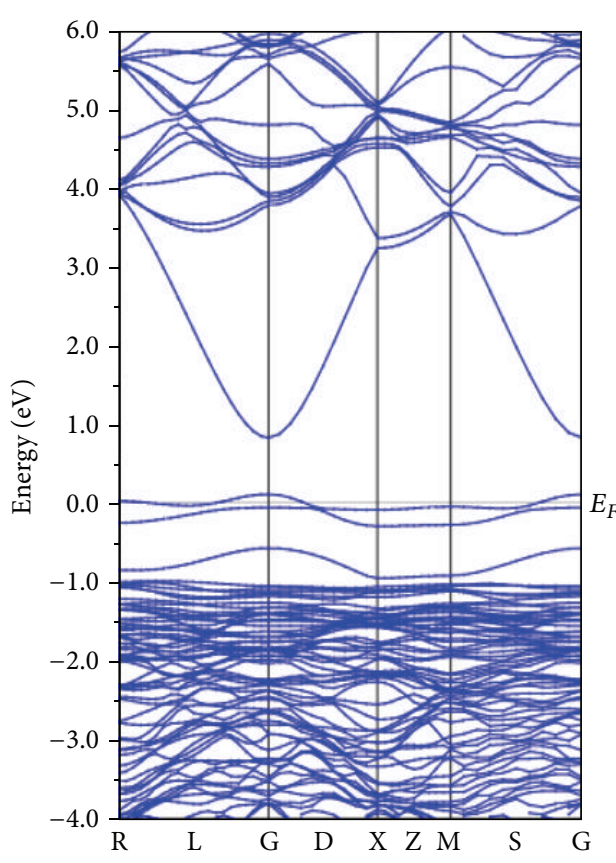

(a)

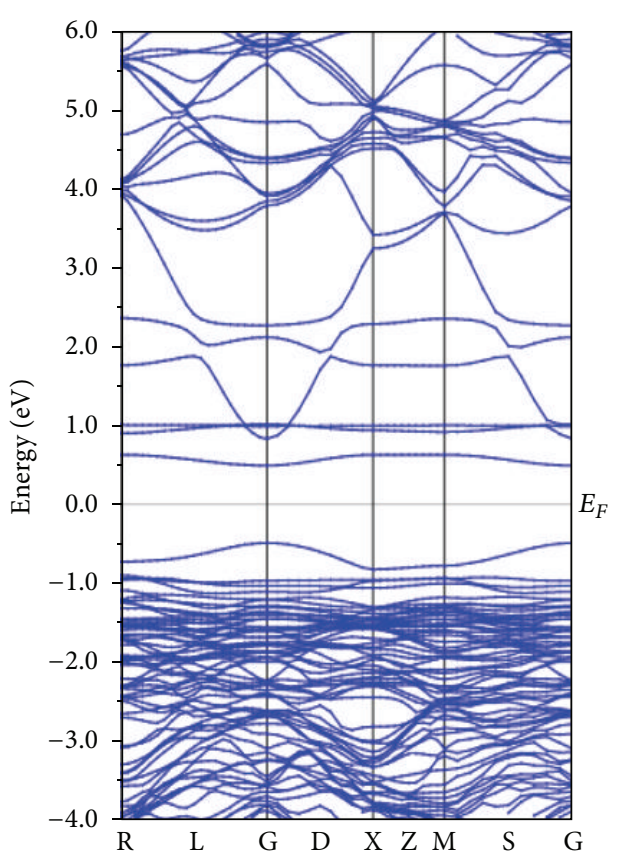

(b)

FIgURE 3: Band structures for supercell: $\mathrm{Sn}_{15} \mathrm{FeO}_{32}$. (a) $x=0.5$ when spin up and (b) $x=0.5$ when spin down.

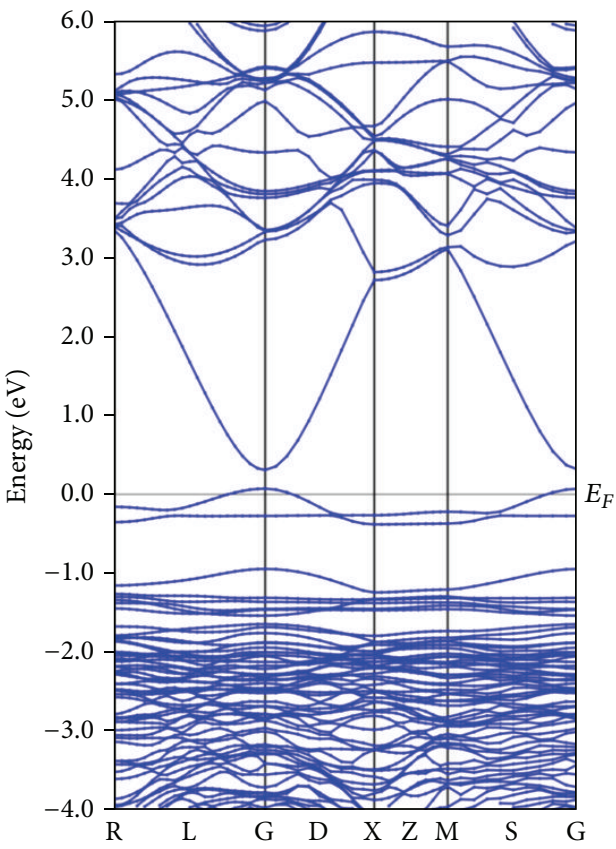

(a)

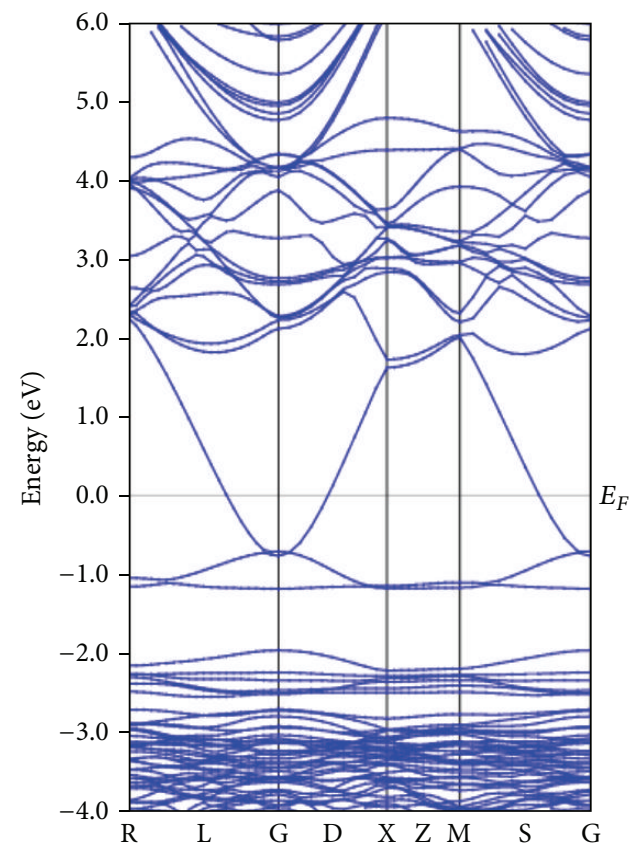

(b)

Figure 4: Band structures for supercell: $\mathrm{Sn}_{15} \mathrm{FeO}_{32}$. (a) $x=1.0$ and (b) $x=1.5$.

The real part $\varepsilon_{1}(\omega)$ of the dielectric function can be evaluated from the imaginary part $\varepsilon_{2}(\omega)$ by the KramerKronig relationship, while the imaginary part $\varepsilon_{2}(\omega)$ has the following expression [29]:

$$
\begin{aligned}
\varepsilon_{2}(\omega)= & \frac{4 \pi^{2}}{m^{2} \omega^{2}} \sum_{V, C} \int_{B Z} d^{3} k \frac{2}{2 \pi}\left|e \cdot M_{c v}(K)\right|^{2} \\
& \times \delta\left[E_{C}(k)-E_{V}(k)-\hbar \omega\right] .
\end{aligned}
$$




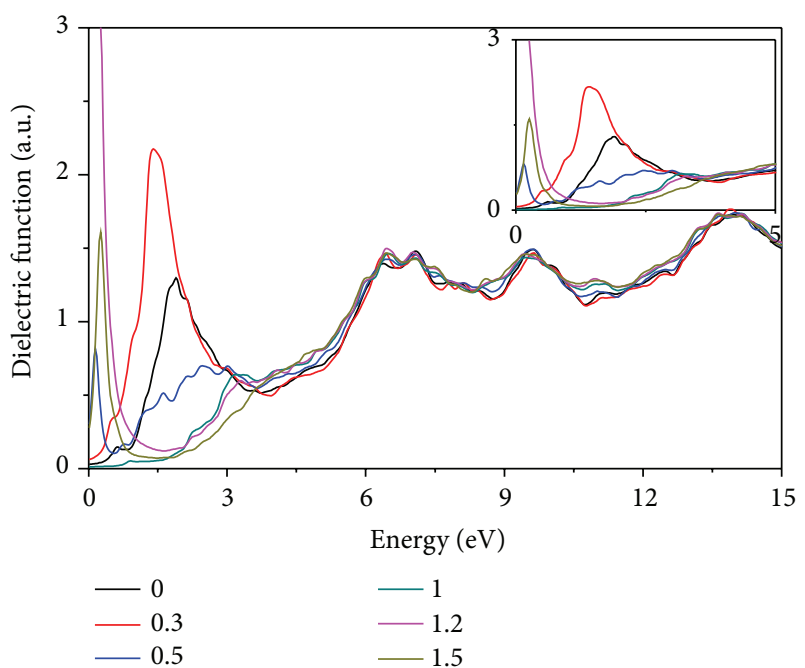

FIGURE 5: The imaginary part of dielectric function $\varepsilon_{2}(\omega)$ of $\mathrm{Sn}_{15} \mathrm{FeO}_{32}$ with electron-injection.

Among this, $\hbar=h / 2 \pi, m$ is the mass of free electrons, $e$ is the charge of free electrons, $\omega$ is the frequency of incident photons, $C$ represents the conduction band, $V$ represents valence band, BZ represents the first Brillouin Zone, and $K$ is the reciprocal vector.

Figure 5 shows the imaginary spectra of optical dielectric function $\varepsilon_{2}(\omega)$, with the number of ions of $0,0.3,0.5,1.0,1.2$, and 1.5. There are three main dielectric peaks ranging from 7.0 to $14.0 \mathrm{eV}$. The first peak at about $7.1 \mathrm{eV}$ should mainly be caused by the transition between $\mathrm{O} 2 \mathrm{p}$ state in the highest valence band and $\mathrm{Sn} 5 \mathrm{~s}$ in the lowest conduction band. The second peak located at about $9.8 \mathrm{eV}$ mainly derives from the transition from $\mathrm{O} 2 \mathrm{p}$ to $\mathrm{Sn} 5 \mathrm{p}$ state, which is reflected by the DOS. The peak at $14.0 \mathrm{eV}$ can be attributed to the combination of the transition between $\mathrm{O} 2 \mathrm{~s}$ and $\mathrm{Sn} 5 \mathrm{~s}$ and that between Fe $3 \mathrm{~d}$ and $\mathrm{Sn} 5 \mathrm{p}$. At the same time, there are some unapparent folded peaks, and they could be attributed to multilevels direct or indirect transition. From 0 to $5.0 \mathrm{eV}$, the peaks changed greatly, which are in consistency with the DOS in Figure 1.

Figure 6 shows the absorption spectra with the number of ions of $0,0.3,0.5,1,1.2$, and 1.5 . In the imaginary spectra of optical dielectric function, three main dielectric peaks exist ranging from 7.2 to $12.0 \mathrm{eV}$, which have no significant differences between various electron-injections, and are in consistency with the dielectric function in Figure 6. As the electron concentrations increase, the overall curves move to the high energy, that is the blue-shift. From 0 to $5.0 \mathrm{eV}$, the peaks changed greatly, the results are consistent with the Ref 30 of $\mathrm{Fe}$-doped $\mathrm{TiO}_{2}$. The spectra become smooth gradually, and the float/drift of the intensities is not apparent, indicating that such optical and electronic devices can work relatively stable.

\section{Conclusions}

In summary, the band structure, the total and partial DOS, and the optical properties of $\mathrm{Sn}_{15} \mathrm{FeO}_{32}$ with electron

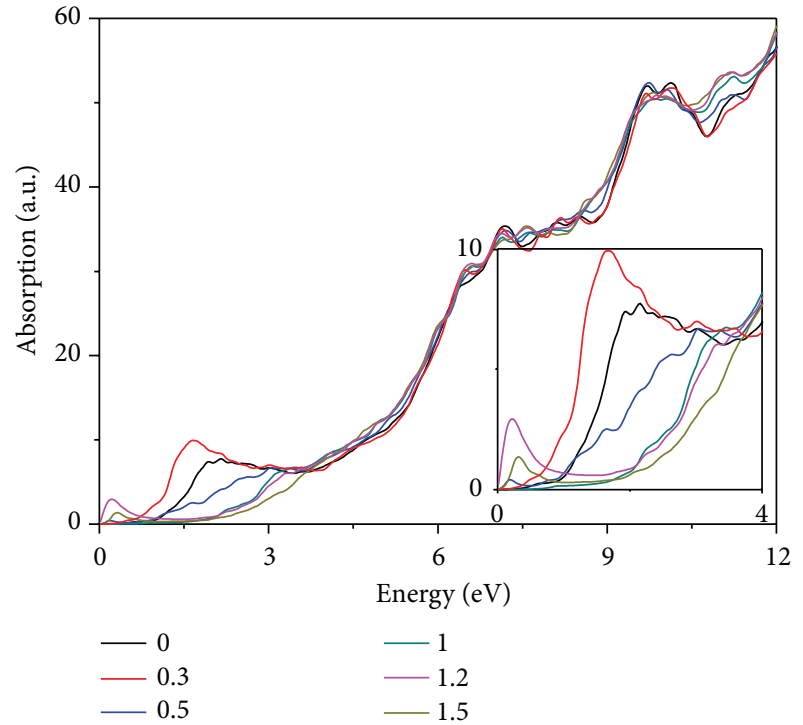

FIGURE 6: Absorption spectra of $\mathrm{Sn}_{15} \mathrm{FeO}_{32}$ with electron-injection.

injection have been investigated by the FP-LAPW method. With the increase of the injected electrons, the conduction band moves to valence band gradually. The $\mathrm{SnO}_{2}$ material shows half-metallic properties when the injected electron is less than 1.0. There exists strong coupling interaction between $\mathrm{Fe}$ atom and $\mathrm{O}$ atom. For the optical properties (the imaginary part of dielectric function, absorption, and reflection), we found that the peaks changed greatly at low energy, the blue shift occurred, and the optical absorption edge increased.

\section{Conflict of Interests}

The authors declare that there is no conflict of interests regarding the publication of this paper.

\section{Acknowledgments}

This work was supported by the National Natural Science Foundation of China (Grant nos. 61172028 and 61076088) and the Natural Science Foundation of Shandong Province (Grant no. ZR2010EL017).

\section{References}

[1] D. Awschalom and M. E. Flatté, "Challenges for semiconductor spintronics," Nature Physics, vol. 3, pp. 153-159, 2007.

[2] S. A. Wolf, D. D. Awschalom, R. A. Buhrman et al., "Spintronics: a spin-based electronics vision for the future," Science, vol. 294, no. 5546, pp. 1488-1495, 2001.

[3] Y.-W. Son, M. L. Cohen, and S. G. Louie, "Half-metallic graphene nanoribbons," Nature, vol. 446, no. 7133, pp. 347-349, 2007.

[4] E. Kan, L. Yuan, and J. L. Yang, "Electron-induced ferromagnetic ordering of Co-doped ZnO," Journal of Applied Physics, vol. 102, no. 3, Article ID 033915, 2007. 
[5] S. Dhar, O. Brandt, A. Trampert et al., "Origin of hightemperature ferromagnetism in $(\mathrm{Ga}, \mathrm{Mn}) \mathrm{N}$ layers grown on $4 \mathrm{H}$ SiC(0001) by reactive molecular-beam epitaxy," Applied Physics Letters, vol. 82, no. 13, pp. 2077-2079, 2003.

[6] J. Schliemann and A. H. MacDonald, "Noncollinear ferromagnetism in (III,Mn)V semiconductors," Physical Review Letters, vol. 88, Article ID 137201, 2002.

[7] Q. Wang, Q. Sun, P. Jena, and Y. Kawazoe, "Ferromagnetic GaNCr nanowires," Nano Letters, vol. 5, no. 8, pp. 1587-1590, 2005.

[8] K. L. Chopra, S. Major, and D. K. Pandya, "Transparent conductors-a status review," Thin Solid Films, vol. 102, no. 1, pp. 1-46, 1983.

[9] A. L. Dawar and J. C. Joshi, "Semiconducting transparent thin films: their properties and applications," Journal of Materials Science, vol. 19, no. 1, pp. 1-23, 1984.

[10] L. Kaplan, A. Ben-Shalom, R. L. Boxman, S. Goldsmith, U. Rosenberg, and M. Nathan, "Annealing and Sb-doping of SnO films produced by filtered vacuum arc deposition: structure and electro-optical properties," Thin Solid Films, vol. 253, no. 1-2, pp. $1-8,1994$.

[11] H. Wang, Y. Yan, Y. S. Mohammed, X. Du, K. Li, and H. Jin, "First-principle study of magnetism in Co-doped $\mathrm{SnO}_{2}$," Journal of Magnetism and Magnetic Materials, vol. 321, no. 5, pp. 337-342, 2009.

[12] A. Kar and A. Patra, "Optical and electrical properties of $\mathrm{Eu}^{3+}$ Doped $\mathrm{SnO}_{2}$ nanocrystals," Journal of Physical Chemistry C, vol. 113, no. 11, pp. 4375-4380, 2009.

[13] E. Zampiceni, E. Bontempi, G. Sberveglieri, and L. E. Depero, "Mo influence on $\mathrm{SnO}_{2}$ thin films properties," Thin Solid Films, vol. 418, no. 1, pp. 16-20, 2002.

[14] R. H. R. Castro, P. Hidalgo, R. Muccillo, and D. Gouvea, "Microstructure and structure of NiO- $\mathrm{SnO}_{2}$ and $\mathrm{Fe}_{2} \mathrm{O}_{3}-\mathrm{SnO}_{2}$ systems," Applied Surface Science, vol. 214, pp. 172-177, 2003.

[15] E. Comini, A. Vomiero, G. Faglia, G. D. Mea, and G. Sberveglieri, "Influence of iron addition on ethanol and CO sensing properties of tin oxide prepared with the RGTO technique," Sensors and Actuators B: Chemical, vol. 115, no. 2, pp. 561-566, 2006.

[16] D. Kotsikau, M. Ivanovskaya, D. Orlik, and M. Falasconi, "Gas-sensitive properties of thin and thick film sensors based on $\mathrm{Fe}_{2} \mathrm{O}_{3}-\mathrm{SnO}_{2}$ nanocomposites," Sensors and Actuators $B$ : Chemical, vol. 101, no. 1-2, pp. 199-206, 2004.

[17] R. Adhikari, A. K. Das, D. Karmakar, T. V. C. Rao, and J. Ghatak, "Structure and magnetism of Fe-doped $\mathrm{SnO}_{2}$ nanoparticles," Physical Review B-Condensed Matter and Materials Physics, vol. 78, no. 2, Article ID 024404, 2008.

[18] H. S. Kim, L. Bi, G. F. Dionne, C. A. Ross, and H. J. Paik, "Structure, magnetic and optical properties, and Hall effect of Co- and Fe-doped $\mathrm{SnO}_{2}$ films," Physical Review B, vol. 77, Article ID 214436, 2008.

[19] X. L. Wang, Z. X. Dai, and Z. Zeng, "Search for ferromagnetism in $\mathrm{SnO}_{2}$ doped with transition metals ( $\mathrm{V}, \mathrm{Mn}, \mathrm{Fe}$, and $\mathrm{Co}$ )," Journal of Physics: Condensed Matter, vol. 20, no. 4, Article ID 045214, 2008.

[20] S. Datta and B. Das, "Electronic analog of the electro-optic modulator," Applied Physics Letters, vol. 56, p. 665, 1990.

[21] I. Robel, M. Kuno, and P. V. Kamat, "Size-dependent electron injection from excited CdSe quantum dots into $\mathrm{TiO}_{2}$ nanoparticles," Journal of the American Chemical Society, vol. 129, no. 14, pp. 4136-4137, 2007.
[22] M. Kohda, Y. Ohno, K. Takamura, F. Matsukura, and H. Ohno, "A spin Esaki diode," Japanese Journal of Applied Physics 2: Letters, vol. 40, no. 12, pp. L1274-L1276, 2001.

[23] K. Schwarz, "DFT calculations of solids with LAPW and WIEN2k," Journal of Solid State Chemistry, vol. 176, no. 2, pp. 319-328, 2003.

[24] K. Schwarz, P. Blaha, and G. K. H. Madsen, "Electronic structure calculations of solids using the WIEN2k package for material sciences," Computer Physics Communications, vol. 147, no. 1-2, pp. 71-76, 2002.

[25] H. J. Monkhorst and J. D. Pack, "Special points for Brillouinzone integrations," Physical Review B: Solid State, vol. 13, no. 12, pp. 5188-5192, 1976.

[26] J. P. Perdew, K. Burke, and M. Ernzerhof, "Generalized gradient approximation made simple," Physical Review Letters, vol. 77, no. 18, pp. 3865-3868, 1996.

[27] W. H. Baur and A. A. Khan, "Rutile-type compounds-IV. $\mathrm{SiO}_{2}$, $\mathrm{GeO}_{2}$ and a comparison with other rutile-type structures," Acta Crystallographica Section B, vol. 27, pp. 2133-2139, 1971.

[28] Z. G. Zhu, R. C. Deka, A. Chutia et al., "Enhanced gassensing behaviour of $\mathrm{Ru}$-doped $\mathrm{SnO}_{2}$ surface: a periodic density functional approach," Journal of Physics and Chemistry of Solids, vol. 70, no. 9, pp. 1248-1255, 2009.

[29] X. D. Zhang, M. L. Guo, C. L. Liu et al., "First-principles investigation of electronic and optical properties in wurtzite $\mathrm{Zn}_{1-x} \mathrm{Mg}_{x} \mathrm{O}$," European Physical Journal B, vol. 62, pp. 417-421, 2008. 

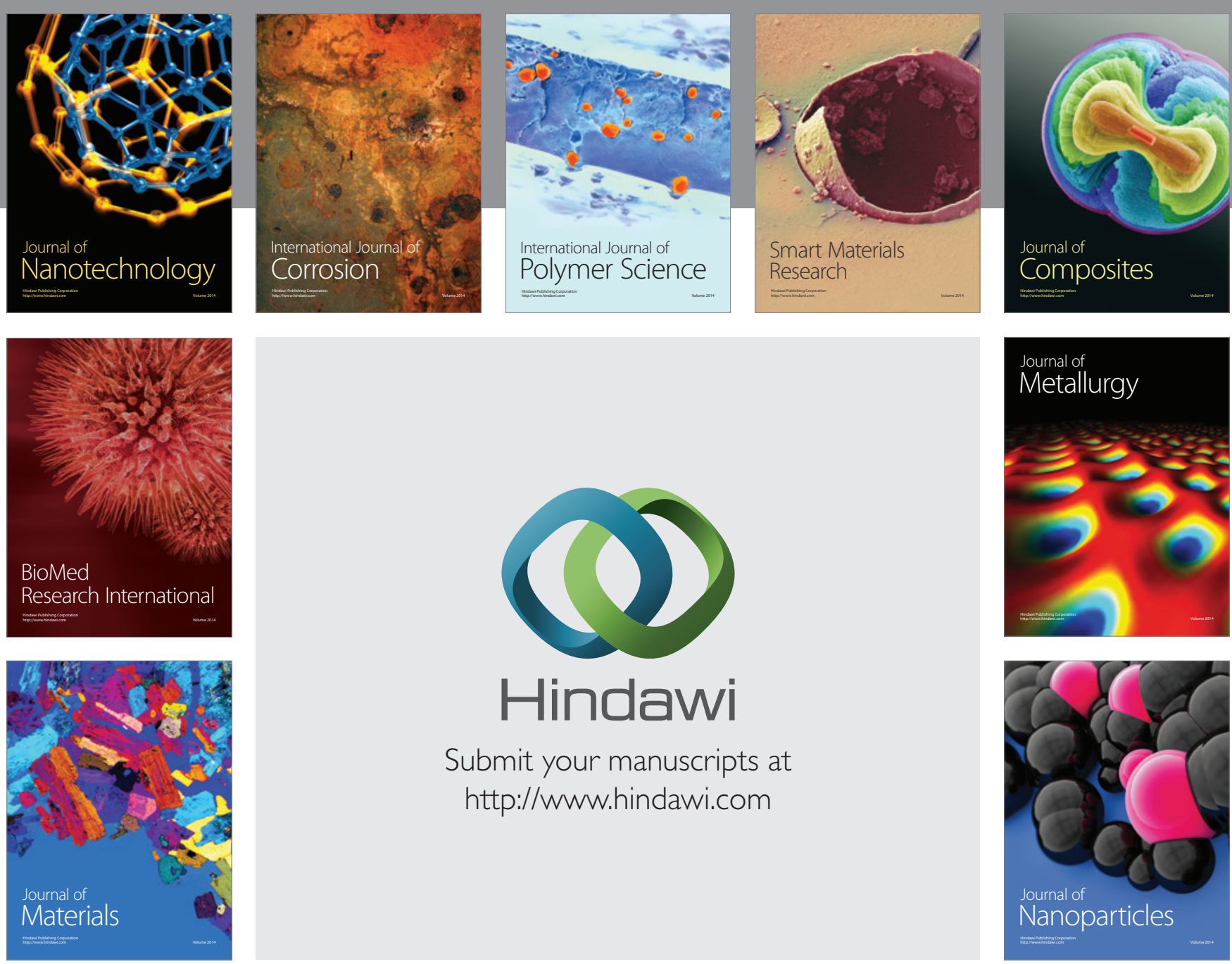

Submit your manuscripts at http://www.hindawi.com
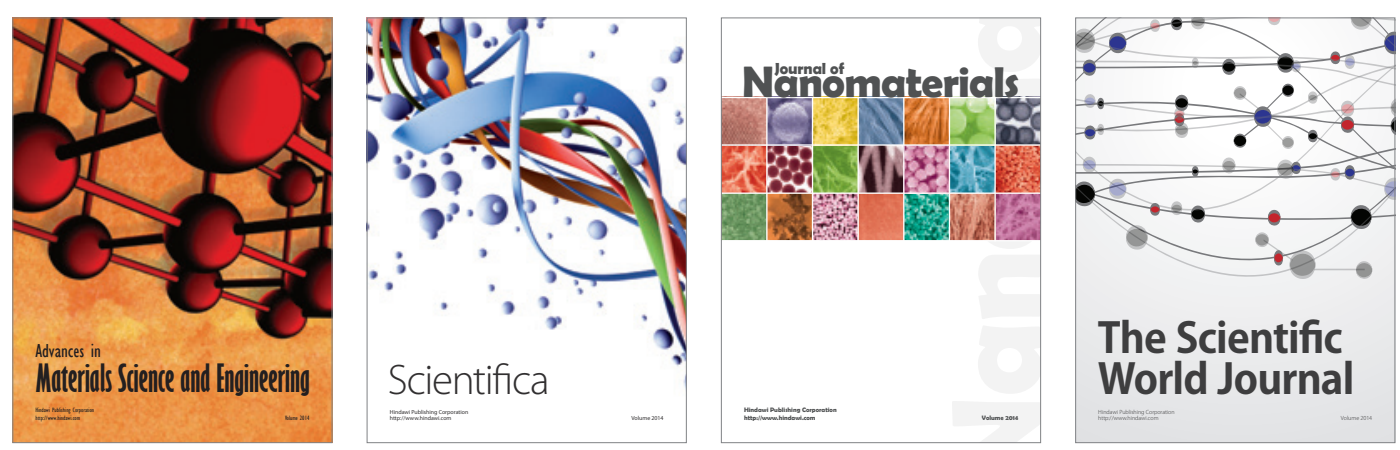

\section{The Scientific World Journal}
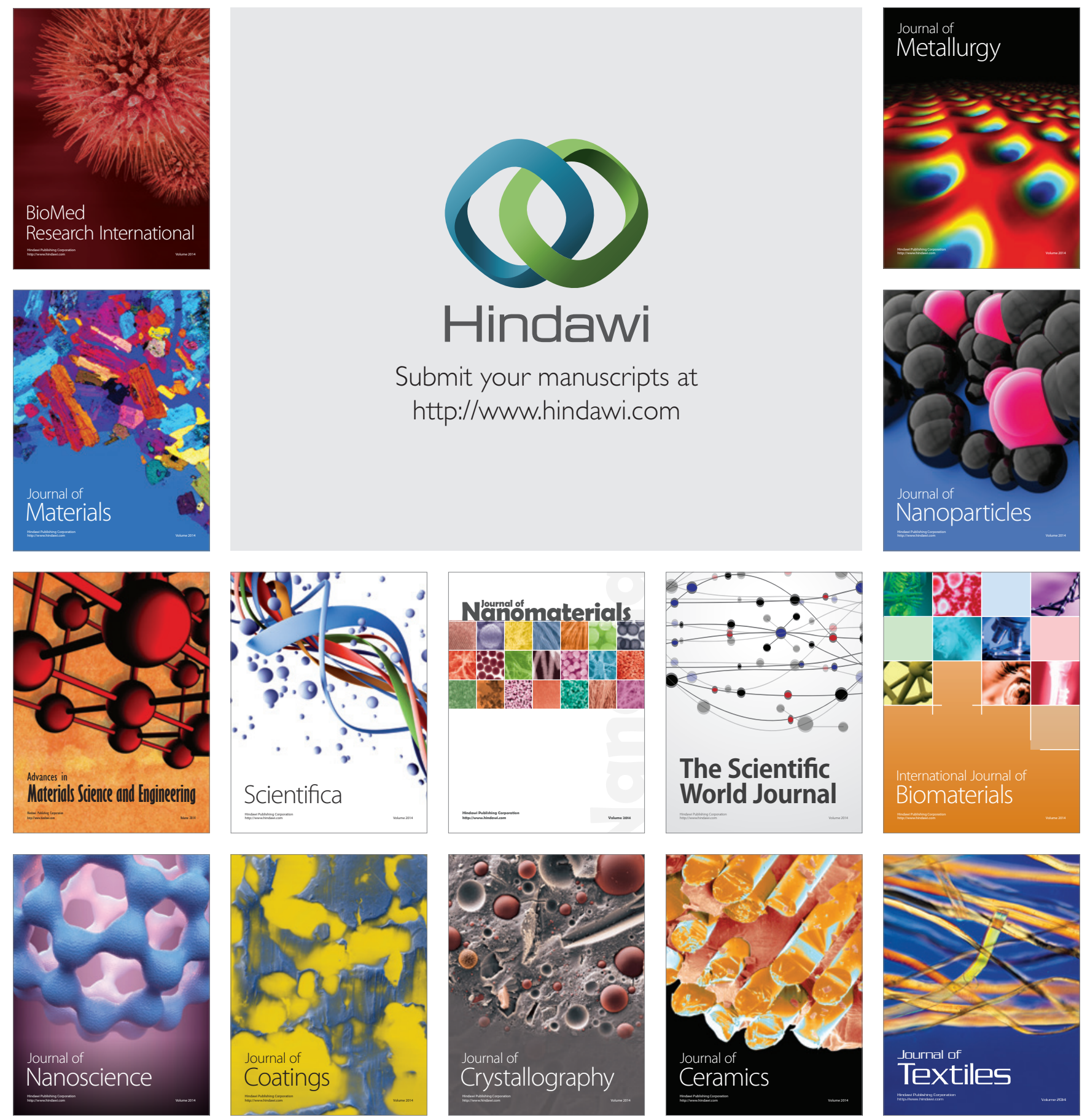